\title{
Contextual Knowledge Sharing in a P2P Network
}

\author{
Philip O'Brien \\ Faculty of Computer Science \\ Dalhousie University \\ Halifax, Nova Scotia B3H 3V6 \\ Email: pobrien@cs.dal.ca
}

\author{
Syed Sibte Raza Abidi \\ Faculty of Computer Science \\ Dalhousie University \\ Halifax, Nova Scotia B3H 3V6 \\ Email:sraza@cs.dal.ca
}

\begin{abstract}
Developing techniques and tools to leverage context and facilitate actions, personalization, tasks, and knowledge has been at the forefront of research in knowledge management with little concern of fully understanding context. Knowledge is created and consumed in a context-i.e. decisions are made, conversations are held, conditions computed, and knowledge is shared with respect to a context.

As knowledge sharing is so critically placed within context, an understanding of this context is essential in developing effective tools. Thus, before further research can be conducted effectively, we must determine the nature and characteristics of context. This paper presents a critical overview of context from multiple perspectives. We will propose a model for elucidating and characterizing context in knowledge sharing, with a practical application to peer-to-peer knowledge sharing networks.
\end{abstract}

\section{INTRODUCTION}

Knowledge is created and consumed in a context-i.e. decisions are made, conversations are held, conditions are computed, and knowledge is shared with respect to the prevailing context. Context, notwithstanding the diversity of its interpretations, is the prime determinant of any knowledge activityas the context varies the nature and application of knowledge sharing varies in order to produce an outcome congruent to the prevailing context. Efficient knowledge sharing is predicated by identifying the right content within a specific context. From a knowledge sharing perspective, context consists of: who created the content, the nature of the content, the knowledgemediated task being performed, when, where and why it was being performed [1]. To date, knowledge sharing research has largely concentrated on selecting the relevant content as per a specific 'static' context. Knowledge sharing research has not adequately expressed the fact that context is a dynamic entity and this dynamism directly impacts the relevance of the content being shared. This brings to relief the need to ascertain the role of context in knowledge sharing activities vis-a-vis context-aware, context-sensitive, and context-driven knowledge management and sharing.

The concept of context is quite pertinent in knowledge management yet it has not been sufficiently investigated and hence not well elaborated [2], [3]. Although many authors use the notion of context, albeit without a clear definition, its meaning, use and formalisms is left to the readers interpretation and understanding [4], [5]. We argue that there is a need to objectively define context in order to investigate its role and impact on various knowledge management activities-i.e. knowledge acquisition, operationalization and sharing would demand different meanings and use of the concept of context as each knowledge management activity pursues a different set of goals and inherently different processes. Context is used in many instances with various definitions or with assumptions that the definition is known. This inconsistency shows that a characterization of context is not known, or perhaps does not exist, with respect to knowledge management. Its importance has often been ignored in general. Disregarding the importance of context can be an oversight as knowing only the problem solution without understanding the problem can lead to misinterpretations [6]. We submit that until we understand the constituents and characteristics of context, the entire scope and functional validity of knowledge-mediated solutions cannot be ascertained. This does not imply that there is a single solution to a $\mathrm{KM}$ problem, rather the assertion is that no valid solution can be rightfully discovered without first considering the prevailing context.

This paper attempts to investigate the conceptualization and characterization of context from different perspectives, beginning with philosophy and anthropology, leading into societal context, linguistic context, and moving to natural language processing, artificial intelligence, context-aware and pervasive computing and knowledge management. Furthermore, we present a context-aware peer-to-peer (P2P) model for knowledge sharing that establishes a context as an interplay between an ontological peer profile and the peer's dynamic dynamic knowledge-mediated tasks that vary with time and in turn modulate the peer's knowledge needs.

The paper is organized into three systematic steps. We first define the specific and general problems in Section II and mention the significance of each. Section III explores the nature and characteristics of context from the three primary approaches found in the literature: defining context, formalizing context, and modeling context. In Section IV the paper presents a preliminary infrastructure describing what has been learned and the future direction of this research. A summary is presented in Section VII.

\section{Problem Statement}

Context has not been well defined in knowledge sharing (KS). In a P2P system, peers share knowledge but the question is "do they also share the same context when sharing knowledge?" This section specifies the problem we address and also 
provides support for a "no" response to this question.

Given a context, how do we determine the affinity between two peers within a knowledge sharing network? In recent $\mathrm{P} 2 \mathrm{P}$ systems, content addressable mechanisms are employed including semantic overlays and distributed hashtables. We argue that a means for supplementing the identity with a set of contextual characteristics is required.

Even when an affinity between peers is established, exchange of appropriate knowledge should be guided by the context surrounding each peer and knowledge resource. Currently, no technique exists to link knowledge resources to a peer in a particular context.

The challenge is to define context and highlight its boundaries, significance and role in a knowledge sharing problem, thus making it distinguishable from the underlying domain knowledge.

\section{UNDERSTANDING CONTEXT}

Our investigation leads to the belief that context related literature can be categorized into three categorical division.

\section{A. Defining Context}

The first step to determining context of knowledge may be to record all meta-knowledge to infer a definition of context from it-using grounded theory research.

For instance, by using a philosophical investigation of context, we note Scharfstein's argument that context is incomprehensibly difficult [7]. Defining context is bound by the context in which we define it. Changing our context changes our interpretation of context. Defining the context in which an interpretation is made is subject to its own context. Interestingly, this description of context implies an infinitely nested space where any context is subject to the interpretation of its parent context. Thus, a definition or complete understanding of context is seemingly incomprehendable.

Pure anthopologists seek not to determine the meaning of context, but its use [8]. Context is thought of in terms of connections between domains. Since delimiting the bounds between domains is difficult, finding discrete bounds on contexts may be futile. Dilley [8] states that to make a connection is to make an interpretation, and as interpretations are contextdependent, context involves making connections.

Holy [9] provides a metaphor for context by comparing it to a frame: context surrounds the situation we are attempting to understand and provides the appropriate resources relevant to achieving that understanding.

Guha [10] states that contexts are objects in a domain about which one can make statements. These objects are rich and partially intangible, thus can never be completely or accurately described. Defining a problem then consists of examining an environment and assessing which context objects are relevant to the desired requirements, goals, and intended solutions.

Ahmadjian discusses knowledge creation as taking place in a $b a$ [3]. $B a$ is described as a context in which interactions take place - physically, virtually, mentally, or socially - to facilitate knowledge creation. More generally, it is a platform upon which knowledge is created, shared and used. All knowledge activities take place in the same $b a$ but are affected differently depending on the task. We later propose to include task as an additional character of context, thus $b a$ is not in itself context but is an environment where we "plug-in" the task, and consequently discover the context.

By explicating $b a$, we get the model proposed by Schmidt et al. [11] in the realm of pervasive computing. This model defines context as the set of surrounding facts that provide meaning to a domain whereby (a) a context describes the environment and situational facts in which a system resides, (b) every context is unique and non-congruent with every other context, (c) every context has a set of relevant features, and (d) each relevant feature has a range of acceptable values determined by the context.

We propose that from the above four points a hierarchical feature space can be developed whereby each feature is derived from a more general feature in the hierarchy. Schmidt, Beigl and Gellersen characterize this concept hierarchy further into a number of levels and nodes.

\section{B. Formalizing Context}

Definitions provide a rudimentary understanding of context. To understand the properties and the operationalization of context, formalisms are proposed.

The first noticable work in formalizing context was proposed by McCarthy [13], [14] and is extended in McCarthy and Buvač [22] as well as Guha [10]. In their work the authors do not attempt to define context, but state only that it exists and influences the environment. The authors provide two functions as the basis for their formalization: ist $(c, p)$ and value $(c, e)$. The first is used to denote a proposition $p$ that is true in a context $c$. The second obtains the value of a term $e$ in the context. Using this formalism, propositions can be expressed in single contexts and their truths inferred in other contexts.

Another contribution of the authors is the notion of lifting axioms that are used to remove contextual-dependance of some proposition or value by a means similar to refactoring. Systematic removal of propositions and values from a context and replacing with predicates that denote their meaning, and not their value (or truthfulness), provides more flexibility to make statements on that context.

Buvač and Mason [16] have introduced the notion of a common context vocabulary. This common vocabulary is well known as a taxonomy and lays the foundation for ontological reasoning in current $\mathrm{KM}$ environments.

Giunchiglia [15] supposes that context is a "theory of the world" [4] which codifies an individuals state relative to that world. Any context $c_{i}$ as viewed by an entity (e.g., individual, organization, computer system) is expressed as a triple: $\left\langle L_{i}, A_{i}, \Delta_{i}\right\rangle$ which describes the language (e.g., natural, or encoded), the set of relevant axioms, and inference mechanisms, respectively. Contexts can be linked by abridging their inference mechanisms and formulating a single, common, language or set of common axioms. 
TABLE I

SUMMARY OF LITERATURE SURVEYED AND PRACTICALITY TO THIS STUDY

\begin{tabular}{|c|c|c|c|c|c|}
\hline Paper & View of Context & $\mathrm{D} / \mathrm{F} / \mathrm{M}$ & Field & Primary Contribution & Practical? \\
\hline Attardi \& Simi [2] & Tangible objects & $\mathrm{F}$ & FOL $(\& \mathrm{KM})$ & Viewpoints & Yes \\
\hline Shoham [12] & Abstract space & $\mathrm{F}$ & Phil & $\begin{array}{l}\text { Every assertion has meaning in } \\
\text { every context; closure axoims }\end{array}$ & No \\
\hline McCarthy [13], [14] & Objects & $\mathrm{F}$ & $\mathrm{AI}$ & Propositional algrbra; Lifting & Yes! \\
\hline Giunchiglia [15] & $\begin{array}{l}\text { Objects as triples } \\
\left\langle L_{i}, A_{i}, \Delta_{i}\right\rangle\end{array}$ & $\mathrm{F}$ & $\overline{\mathrm{DB}}$ & Bridging inference mechanisms & No \\
\hline Guha [10] & Objects & $\mathrm{F}$ & $\mathrm{AI}$ & Micro-theories & Yes \\
\hline Buvac \& Mason [16] & $\begin{array}{l}\text { Objects with } \\
\text { properties }\end{array}$ & $\mathrm{F}$ & Phil & $\begin{array}{l}\text { Need for a common context } \\
\text { vocabulary }\end{array}$ & Yes \\
\hline Akman \& Surav [4] & $\begin{array}{l}\text { Iterative } \\
\text { process }\end{array}$ & $\mathrm{F}$ & $\mathrm{AI}$ & $\begin{array}{l}\text { Knowledge task; } \\
\text { Feedback }\end{array}$ & Yes \\
\hline Scharfstein [7] & - & $\bar{D}$ & Phil & $\begin{array}{l}\text { Argues that context is too difficult to } \\
\text { comprehend; no outermost context }\end{array}$ & No \\
\hline Otzturk \& Aamodt [17] & $\begin{array}{l}\text { Related concepts } \\
\text { with properties }\end{array}$ & $\bar{M}$ & KM & $\begin{array}{l}\text { Use of ontologies for } \\
\text { representation }\end{array}$ & Yes \\
\hline Dilley [8] & $\begin{array}{l}\text { Relations between } \\
\text { domains }\end{array}$ & $\bar{D}$ & Anth & $\begin{array}{l}\text { Context involves making } \\
\text { connections }\end{array}$ & Yes \\
\hline Holy [9] & $\begin{array}{l}\text { Frame of } \\
\text { interest/relevance }\end{array}$ & $\bar{D}$ & Anth & $\begin{array}{l}\text { All aspects of context are relevant } \\
\text { with varying degrees; context is a } \\
\text { frame of interest/relevance }\end{array}$ & Yes \\
\hline Halpin [18] & $\begin{array}{l}\text { Discrete objects } \\
\text { and relations }\end{array}$ & $\bar{M}$ & $\mathrm{DB}$ & $\begin{array}{l}\text { Graphical representations of objects, } \\
\text { their properties, and relations }\end{array}$ & No \\
\hline Henricksen et al. [19] & $\begin{array}{l}\text { Discrete objects } \\
\text { (data) }\end{array}$ & $\mathrm{D} / \mathrm{M}$ & $\begin{array}{l}\text { Pervasive } \\
\text { computing }\end{array}$ & UML Style notation & No \\
\hline Chtcherbina \& Franz [20] & $\begin{array}{l}\text { Discrete objects } \\
\text { with dependencies }\end{array}$ & $\mathrm{M}$ & $\begin{array}{l}\text { Context aware } \\
\text { computing }\end{array}$ & $\begin{array}{l}\text { Hierarchical dependencies between } \\
\text { contextual facts }\end{array}$ & No \\
\hline $\begin{array}{l}\text { Kwan \& } \\
\text { Balasubrimanian [1] }\end{array}$ & $\begin{array}{l}\text { Organizational } \\
\text { process }\end{array}$ & $\bar{D}$ & $\begin{array}{l}\mathrm{KM} / \\
\text { organizational }\end{array}$ & $\begin{array}{l}\text { Human and tasks elements of } \\
\text { context }\end{array}$ & Yes \\
\hline Ahmadjian [3] & Abstract space & $\mathrm{D} / \mathrm{M}$ & Organizational & The "Ba" & No \\
\hline Kahol et al. [21] & Tacit objects & $\bar{M}$ & $\begin{array}{l}\text { Cognitive } \\
\text { psychology }\end{array}$ & $\begin{array}{l}\text { Multiple interrelated } \\
\text { contexts }\end{array}$ & Yes \\
\hline Schmidt et al. [11] & Explicit facts & $\bar{M}$ & $\begin{array}{l}\text { Pervasive } \\
\text { computing }\end{array}$ & $\begin{array}{l}\text { Context history; context has a set of } \\
\text { relevent features, each with a range } \\
\text { of acceptable values }\end{array}$ & Yes \\
\hline
\end{tabular}

Attardi and Simi [2] introduced the notion of viewpoint. Following first-order logic (FOL) whereby context is a set of reified truths, as defined by the agent engaging in an activity, viewpoints are then constructed and used to "express varieties of relativized truth" [4]. The authors also provide the first formal expression of knowledge in context. Here, belief is an axiom provable by an agent, $g$, from that agent's viewpoint, $\operatorname{Bel}(g, A)=i n\left(A, v p_{g}\right)$. Truth is anything provable in the real world, thus making knowledge the set of true beliefs of a set of axioms from an agents viewpoint:

$$
K(g, A)=\bigcup_{A} \operatorname{Bel}(g, A) \wedge \operatorname{True}(A)
$$

Notwithstanding the utility of these approaches, it may be prudent to extend beyond formalizing context towards modeling context.

\section{Modeling Context}

While it is argued that the difference between formalizing context and modeling context is moot [1], [4], [5], a distinction is drawn here. Formalization alludes to mathematically, logically, or abstractly describing context for philosophical considerations. Modeling context, in this paper, involves creating frameworks or relational models of how context interacts with elements of interest in an environment.
Strang and Linnhoff-Popien [5] provide a survey on context from a ubiquitous computing perspective. A data structure for modeling context is acquired through key-value pairs [5]. This structure models discrete sets of attributes identified in an environment that can be elicited and codified. Values are stored as associations to keys-unique accessors to the value.

More advanced schemes attempt to model context as recursive hierarchies of markup [5], extending traditional hierarchical models like that of SGML or XML to compensate for the lack of semantic relations expressible in XML. As a result, many have reached the expressiveness of $\mathrm{RDF}(\mathrm{S})$.

Another approach to context modeling in the markup scheme is the Pervasive Profile Description Language (PPDL) [20]. This model encodes dependencies within contextual information. This symbolic hierarchy follows from the physical hierarchy of the representation modality; namely, XML/RDF.

Kahol et al. [21] describes context much as we might expect in knowledge management, but slanted toward psychology. It is the set of things a user carries with them, together with the inherent variables in an environment. Actions of a person are guided by their context in which they act. From this angle, context is seen as largely tacit. It cannot be influenced directly but only through time.

Finally, context from an organization perspective is modelled as discrete units that can be expressed and are unchang- 
ing. For an established organization, context is deterministic and nearly static, subject only to manual, explicit change from agents of the environment [1].

The literature we have surveyed has been categorized into: (1) defining context, (2) formalizing context, and (3) modeling context. The viewpoints of each paper are summarized in Table $\mathrm{I}^{1}$.

\section{Building a ConteXt-Based Knowledge Sharing FRAMEWORK}

Based on the literature survey provided above, this section outlines a preliminary model of context that is relevant towards knowledge sharing networks.

Our context-sensitive knowledge sharing framework defines context as the set of semantically related propositions, having a set of features each with a range of acceptable values, that are dynamic and derivable from a situation and are relevant to varying degrees from a given perspective for involved actors engaged in a common task, which evolves progressively over time. We explain the definition's constituent elements below.

1) set of semantically related propositions: Propositions hold in a subset of contexts subject to the above constraints. McCarthy [13] and Guha [10] have shown that these contexts can be related via lifting mechanisms. The semantic relationship was demonstrated in Kahol et al. [21]. Propositions having a shared truth are interpreted to be related based on meaningful interconnections of their meanings in a context.

2) having a set of features each with a range of acceptable values: Features of a context-i.e. elements of a proposition that characterize that proposition within a domain — cannot be arbitrarily defined. Context is described as a set of features that depict distinct concepts where each feature has a range of acceptable values [11].

3) that are dynamic and derivable from a situation: Context is a dynamic product arising from the feedback solicited from other actors within the knowledge environment (or intersecting contexts). Feedback is essential if determinants of a context, such as an actors profile, that contribute the context's dynamism are to be modeled [4].

4) and are relevant to varying degrees: Contexts are inherently relevant, with varying degrees [12]. Factors within an environment that do not influence decisions, or influence them to a degree less than some desired threshold, are said to be "outside" the context of interest-the frame of relevance [9]. This frame can be visualized as a discrete-edge boundary to the context of interest where those propositions inside the boundary are relevant to a high degree. Visualizing context as regions of an $n$-dimensional space, where $n$ is the number of relevant features of the environment, many observations can be made. Regions that intersect (i.e. have propositions that hold true in each) are said to be semantically related.

\footnotetext{
${ }^{1}$ The fourth column of Table I indicates the category to which the paper belongs: (D)efinition, (F)ormalization, and (M)odeling
}

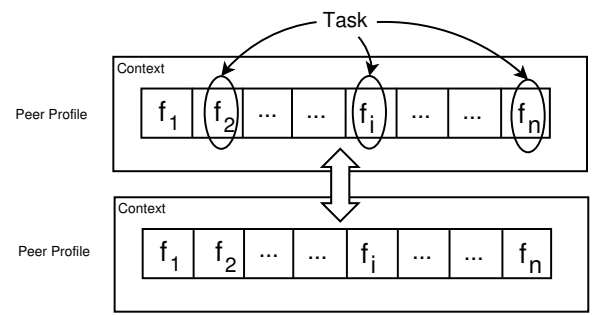

Fig. 1. Context building in the knowledge-sharing process. The task is used to selected the most relevant features of the profile that will influence the context. Here, the feature set $\left\{f_{2}, f_{i}, f_{n}\right\}$ is selected from each profile and used in affinity matching.

5) from a given perspective: Propositions are only relevant given ones perspective [2]. Truths that hold for an actor of a task may not hold for others in the same task. This observation occurs because of the discrepancy between the propositions' semantics-those other propositions that the actor holds to be true in their viewpoint. Viewpoint is the way in which one views a context. It is how the set of features that populate a context are interpreted and reasoned upon. Continuing from the example above, viewpoint can be seen as the angle taken to view an $n$-dimensional context space.

6) for involved actors: Knowledge is not exchanged, nor indeed meaningful, without considering the human element [1]. The knowledge actor defines how knowledge is shared and how the context for that actor is represented in the actor's profile. A knowledge sharing system is both driven and guided by the use of an actor. The context of a KS system is impacted by the context surrounding the actor.

7) engaged in a common task: The differentiating factor between traditional Information Retrieval (IR) systems and a KM system is the task [1], [3]. A knowledge sharing system is certainly dependant on the task being conducted. The task changes and is followed by other tasks by other actors. This time-oriented workflow model is of paramount concern in a context-characterizing model.

8) and which evolves progressively over time: Context is progressive for a task. It changes even during a task. Context is intrinsic but always changing for a user. A user will always have knowledge which will grow, shrink, and adapt to a knowledge task. In knowledge sharing this takes the form of a changing peer profile. Patterns develop in the profile and tracking these patterns allows generation of a context history [11] which can be used for future decision-making, personalization, and validation [17].

\section{Matrix Augmentation for Knowledge Profiles}

Preliminary work indicates representing knowledge as a weighted task-feature relevance matrix provides fast, accurate and flexible access to dynamic context in a network. This approach has not been considered when using task knowledge to collect relevant features from a profile for matching.

The differentiating factor in knowledge networks is the notion of task. Using the task as a type of projection for selecting the set of relevant features from the matrix allows us 


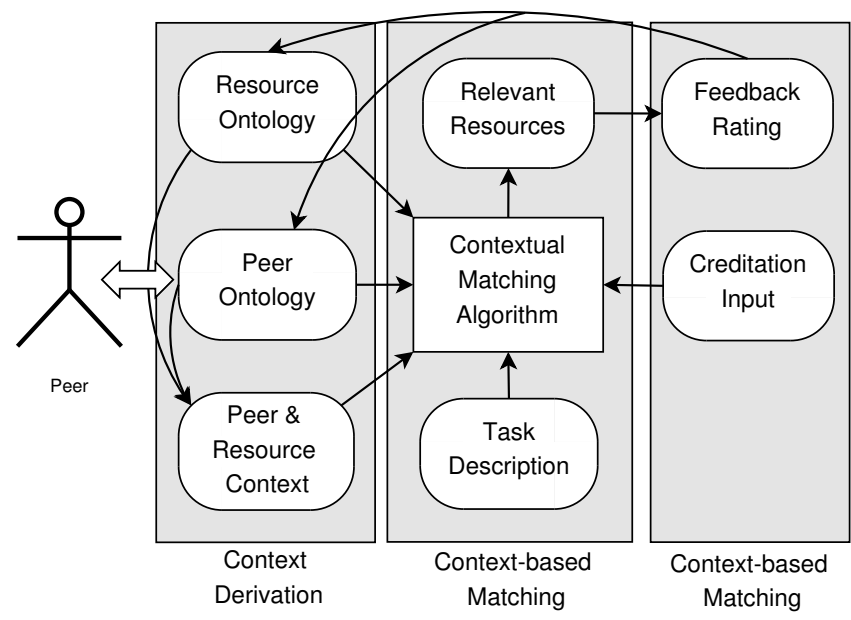

Fig. 2. Experiential and collaborative context feedback process in knowledge sharing

to compute a weighted sum of features that impact the outcome of a decision in a P2P knowledge sharing network. With each peer modeled as an ontological peer profile, the set of profile features can be expressed as $F_{p}$. There are a subset of profile features that are relevant to a task, thus $F_{t} \subset F_{p}$. Each feature of $F_{t}$ impacts the formation of the context to some degree and the impact is represented as the feature's weight. Features irrelevant to a task are given a weight of 0 .

The matrix is composed of the $n$ features of a peer profile and the $m$ identified tasks of a knowledge activity. The relevance of feature $i$ for a task $j$ is denoted by $w_{i, j}$ in the matrix. The contextual relevance of a peer profile to a task is denoted $\sum_{i=1}^{n} w_{i, j}$. When a modification is made to the weights of the matrix, the target task-feature pair, $w_{t, j}$, becomes $w_{t, j}+c$ where $c$ is the impact change, positive or negative. As contexts are interwoven, all other weights are adjusted according to a task stabilization rule

$$
\forall f_{x} \in F_{p} \mid x \neq i \Rightarrow w_{x, i}+\frac{c}{\left|F_{p}\right|-1}
$$

The weights of the features for a task are relative to all other features, thus the sum of the weights for each row must be a consistent maximum value, for example 1.0 or $n$.

\section{A. Proposed Model}

A model is needed to demonstrate how context is determined and influenced by the domain, knowledge task, and knowledge user. Figure 1 illustrates our approach to determining the relevant knowledge resources within a P2P network based on peer profiles and a contextual matching scheme.

Our model takes a set of domain features and uses the knowledge task as a selector which defines the feature set relevant to the requirements, needs, and goals of the task. These features are matched with the peer ontology to define a context for that peer, for that task, and for that domain. Context is derived from the state of a profile partnered with other profiles and document contexts over time. As a profile changes the context surrounding it changes. Persistent changes or trends over time result in changes to the profile to manifest those trends and patterns. This dynamic oscillation and constant evolution will result in more accurate and representative knowledge sharing activities in the KS system.

For example, two peer profiles are represented using both an ontology and a matrix. A row of each peer's matrix is selected based on the task of each peer. If a match is to be determined from the perspective of a local peer (according to the fifth point of Section IV) its features are placed in descending order according to significance. The weights of other peers are computed against the same feature's weight of the local peer, in the order specified. The results are summed for a similarity value relative to other peers.

Feedback is a device for modifying how this match is done and weights of the features deemed significant in finding a contextual match. Feedback in this model occurs in two ways:

1) Feedback from other entities: This feedback modifies a peer's profile and comes in the form of ratings indicating the level of confidence or level of acceptance of the peer's contributions to the network/community. It also comes as ratings of the knowledge resources in the peer's repository which then is a reflection of the peer's accrediation.

2) Experiential feedback from outcomes of tasks and contexts into the context of the task to be used again later: When a task is completed the results are used to update the context for the next time in which the task is engaged.

A rating feature in the peer and task profiles might be a collaborative filter for feedback from the community to change the values of items in the item set of the domain, shown at the top of Figure 1.

The experiential feedback would provide the context with new information in light of the outcome of a task. This new knowledge would be available the next time this context is encountered. This provides for an ever changing context which identifies with the observed changes in knowledge tasks experienced over time.

The above mentioned process is shown in Figure 2. User interaction causes changes in the peer profile and the observed context. These changes result in a recomputation of the similarity of the local peer's context matrix to all other peers of the network. Using each peer's current task, a list of resources are determined to "match" the peer's current context. This list is reported back to the user who then has the opportunity to provide feedback on the knowledge resource ontology as well as the peer ontology and profile.

\section{Context-based Peer-to-Peer Knowledge SHARING NETWORKS}

Frameworks based on context-aware and pervasive computing strategies [23] rely on explicit and tacit feedback from users of the system.

When knowledge actors require documents from a system, having insight into the interests of the requisite actor may allow for more accurate retrieval to occur. Abidi and Pang [24] propose using two ontologies: a peer ontology used to 


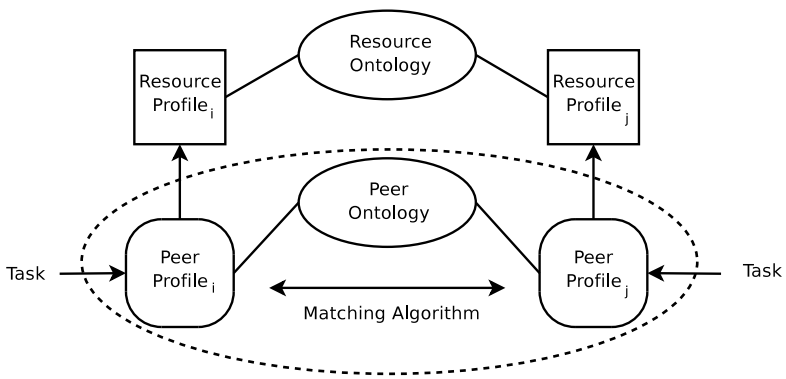

Fig. 3. Model of P2P knowledge sharing network using context. Context surrounds the ontological peer profile matching algorithm and the peer and document profiles. The task collects relevant features from the profiles based on impact weights from the task-feature relevance matrix. The profiles are feature-based as shown in Figure 1

match interests of peers in a $\mathrm{P} 2 \mathrm{P}$ network, and a document ontology used to determine affinity between those documents.

The peer ontology is used to measure the degree of shared interest between peers in a network on a given topic. It is used to determine if one peer has resources of interest to the other, based on the context surrounding each peer. Peers which exhibit strong semantic relationships (on multiple topics, or great depth of a few topics) are located within proximity of each other in a semantically addressable overlay network. Resources are listed by each peer as being of interest or not. Such a dichotic classification is often used to indicate what is interesting and not interesting to the user [25]. Alternatively, only interesting topics could be included and anything not in the list could be deemed as "not interesting". This singular classification, however, is too restrictive as unclassified items are assumed to be of no interest.

In our work, when the similitude of two peers is determined to be high, resources are shared that are of the same type as those with which the requesting peer has exhibited interest. At this stage we transcend the context to be above the contextual mapping of the peer model to the domain model. The context, as shown in Figure 3, surrounds the peer profiles and the matching algorithm. We have added the context to what is known of the peer as being supplementary to the peer profile.

\section{SUMMARY}

This paper presents a survey of how context has been defined from a number of perspectives. We have stressed the importance of context in knowledge sharing and have presented a conceptual overview of how the identified elements of context can be used to formulate a context-sensitive $\mathrm{P} 2 \mathrm{P}$ knowledge sharing framework. The implementation of the $\mathrm{P} 2 \mathrm{P}$ system is in progress and will be followed by evaluation studies that will be reported in subsequent publications.

\section{ACKNOWLEDGEMENTS}

This research is funded in part by the National Sciences and Engineering Research Council (NSERC) of Canada.

\section{REFERENCES}

[1] M. M. Kwan and P. Balasubramanian, "KnowledgeScope: managing knowledge in context," Decision Support System, vol. 35, no. 4, pp. 467-486, 2003.

[2] G. Attardi and M. Simi, "A formalization of viewpoints," Fundamenta Informaticae, vol. 23, no. 5, pp. 149-174, 1995.

[3] C. L. Ahmadjian, Hitotsubashi on Knowledge Management. Singapore: John Wiley \& Sons (Asia) Pte Ltd, 2004, ch. 8: Inter-organization Knowledge Creation: Knowledge and Networks, pp. 227-245.

[4] V. Akman and M. Surav, "Steps toward formalizing context," AI Magazine, vol. 17, no. 3, pp. 55-72, March 1996.

[5] T. Strang and C. Linnhoff-Popien, "A context modeling survey," in First International Workshop on Advanced Context Modelling, Reasoning And Management (UbiComp-2004), September 2004.

[6] A. K. Wensley and A. Verwijk-O'Sullivan, Knowledge Horizons: The Present and the Promise of Knowledge Management. Woburn, MA, USA: Butterworth-Heinemann, 2000, ch. Tools for Knowledge Management, pp. 113-129.

[7] B. A. Scharfstein, The Dilemma of Context. New York, NY, USA: New York University Press, 1989

[8] R. Dilley, The Problem of Context, ser. Methodology and History in Anthropology. New York, USA: Barghahn Books, 1999, vol. 4.

[9] L. Holy, The Problem of Context, ser. Methodology and History in Anthropology. New York, NY, USA and Oxford, UK: Barghahn Books, 1999, vol. 4, ch. 1: Contextualization and Paradigm Shifts.

[10] R. V. Guha, "Contexts: A formalization and some applications," Ph.D. dissertation, Stanford University, 1991.

[11] A. Schmidt, M. Beigl, and H. Gellersen, "There is more to context than location," Computers \& Graphics Journal, vol. 23, no. 6, pp. 893-902, December 1999.

[12] Y. Shoham, Varieties in Context. Boston, MA, USA: Academic Press, 1991, pp. 393-408.

[13] J. McCarthy, Formalizing Common Sense: Papers by John McCarthy. Norwood, NJ, USA: Ablex Publishing Corporation, 1990.

[14] — , "Notes on formalizing contexts," in Proc. of the Fifth National Conference on Artificial Intelligence, T. Kehler and S. Rosenschein, Eds. Los Altos, CA, USA: Morgan Kaufmann, 1987, pp. 555-560. [Online]. Available: citeseer.ist.psu.edu/mccarthy93notes.html

[15] F. Giunchiglia, "Contextual reasoning," Epistemologia, vol. 16, pp. 345364, 1993.

[16] S. Buvač and I. A. Mason, "Propositional logic of context," in Proc. of the 11th National Conference on Artificial Intelligence, 1993, pp. 412-419.

[17] P. Ötztürk and A. Aamodt, "Towards a model of context for case-based diagnostic problem solving," in Proc. of the Interdisciplinary Conference on Modeling and Using Context, February 1997, pp. 198-208.

[18] T. A. Halpin, Information Modeling and Relational Databases: From Conceptual Analysis to Logical Design. San Francisco, CA, USA: Morgan Kaufman Publishers, April 2001.

[19] K. Henricksen, J. Indulska, and A. Rakotonirainy, "Generating context management infrastructure from high-level context models," in Industrial Track Proceedings of the Fourth International Conference on Mobile Data Management, January 2003, pp. 1-6.

[20] E. Chtcherbina and M. Franz, "Peer-to-peer coordination framework (P2PC): Enabler of mobile ad-hoc networking for medicine, business, and entertainment," in Proc. of International Conference on Advances in Infrastructure for Electronic Business, Education, Science, Medicine, and Mobile Technologies on the Internet, January 2003.

[21] K. Kahol, P. Tripathi, T. McDaniel, and S. Panchanathan, "Modeling context in haptic perception, rendering and visualization," in $A C M$ International Workshop on Multimedia Information Systems, 2005.

[22] J. McCarthy and S. Buvač, "Formalizing context (expanded notes)," Computing Natural Language, pp. 13-48, 1997.

[23] P. Amann and G. Quirchmayr, "Foundation of a framework to support knowledge management in the field of context-aware and pervasive computing," in Proc. of the Conferences in Research and Practice in Information Technology, vol. 21. Australian Computer Society, 2003.

[24] S. S. R. Abidi and X. Pang, "Knowledge sharing over P2P knowledge networks: A peer ontology and semantic overlay driven approach," International Conference on Knowledge Management, December 2004.

[25] S. E. Middleton, D. C. De Roure, and N. R. Shadbolt, "Capturing knowledge of user preferences: Ontologies in recommender systems," in International Conference on Knowledge Capture, 2001, pp. 100-107. 